\title{
Lessons from Launching the Diabetes Prevention Program in a Large Integrated Health Care Delivery System: A Case Study
}

\author{
Colin D. Rehm, PhD, MPH, ${ }^{1,2}$ Melinda E. Marquez, MPH, CHES, ${ }^{1}$ Elizabeth Spurrell-Huss, MPH, MSW, \\ Nicole Hollingsworth, EdD, MCHES, and Amanda S. Parsons, MD, MBA ${ }^{1,3}$
}

\begin{abstract}
There is urgent need for health systems to prevent diabetes. To date, few health systems have implemented the evidence-based Diabetes Prevention Program (DPP), and the few that have mostly partnered with community-based organizations to implement the program. Given the recent decision by the Centers for Medicare \& Medicaid Services to reimburse for diabetes prevention, there is likely much interest in how such programs can be implemented within large health systems or how community partnerships can be expanded to support DPP implementation. Beginning in 2010, Montefiore Health System (MHS), a large health care system in the Bronx, NY, partnered with the Young Men's Christian Association (YMCA) of Greater New York to deliver the YMCA's DPP. Over 4 years, 1390 referrals to YMCA's DPP were made; 287 participants attended $\geq 3$ classes, and average weight loss was $3.4 \%$. Because of increased patient demand and internal capacity, MHS assumed responsibility for DPP implementation in May 2015. Fully integrating the program within the health system took 5-6 months, including configuring electronic health record templates/reports, hiring a coordinator, and creating clinical referral workflows/training guides. Billing workflows were designed for risk-based contracts. In the first 11 months of implementation, 1277 referrals were made, and referrals increased over time. Twenty-four class cycles were initiated, and 282 patients began attending classes. Average weight loss among 61 graduates from the Summer/Fall 2015 wave of MDPP classes was 3.8\%. Additional opportunities for expansion include training allied health staff, providing patient incentives, increasing master trainer capacity, offering DPP to employees, and securing reimbursement.
\end{abstract}

Keywords: diabetes, diabetes prevention, chronic disease

\section{Background}

$\mathbf{T}$ YPE 2 DIABETES MELLITUS (T2DM) represents a major population health burden in the United States, doubling in prevalence from 1980 to 2014 . $^{1}$ Diabetes is the seventh leading cause of death in the United States and is a key risk factor for cardiovascular and cerebrovascular disease. ${ }^{2-4}$ As of 2013, diabetes accounted for about 3.5\% of disability-adjusted life years in the United States, a burden comparable to that of Alzheimer's (3.3\%) and depression (3.7\%). ${ }^{5}$ Although prediabetes is a generally symptom-free state defined by higher than normal, but lower than diabetic, blood glucose concentrations, the body may already experience chronic inflammation. ${ }^{6}$ In- dividuals with prediabetes have a substantially elevated risk of developing diabetes, with those closer to the diagnostic threshold deemed to be in need of closer monitoring based on being at higher risk. ${ }^{7}$ The annualized risk of developing T2DM among those with prediabetes is between $5 \%$ and $10 \%$, and $70 \%$ of individuals with prediabetes will go on to develop T2DM in their lifetime. ${ }^{4}$ Recent estimates from a nationally representative survey indicate that $36.5 \%$, or 83 million, US adults are living in a prediabetic state. ${ }^{8}$ The percentage of US adults with prediabetes has increased 1.24-fold from 19992002 to $2007-2010$ (from $29.2 \%$ to $36.2 \%$ ). ${ }^{9}$

The Diabetes Prevention Program (DPP) trial showed that lifestyle changes could reduce the incidence of T2DM by

\footnotetext{
${ }^{1}$ Office of Community \& Population Health, Montefiore Health System, Bronx, New York.

Departments of ${ }^{2}$ Epidemiology and Population Health and ${ }^{3}$ Family \& Social Medicine, Albert Einstein College of Medicine, Bronx, New York

(C) Colin D. Rehm et al 2017; Published by Mary Ann Liebert, Inc. This article is available under the Creative Commons License CC-BY-NC (http://creativecommons.org/licenses/by-nc/4.0). This license permits non-commercial use, distribution and reproduction in any medium, provided the original work is properly cited. Permission only needs to be obtained for commercial use and can be done via RightsLink.
} 
$58 \%$ over an average of 2.8 years of follow-up. ${ }^{10}$ The trial also found that lifestyle changes performed better than treatment with metformin alone. The Centers for Disease Control and Prevention (CDC) used the results of the trial to develop a curriculum in 2010 for uptake in health care and community settings. ${ }^{11}$ As of spring 2016, 800 programs are pending recognition by the $\mathrm{CDC}$ and 56 have received recognition. Numerous nonprofit agencies have made an effort to scale the program nationwide, the most notable being the Young Men's Christian Association (YMCA) and the American Association of Diabetes Educators. ${ }^{12,13}$ Relatively low cost and scalable computer and web-based versions of the DPP, some incorporating human coaching and others being fully automated, have rapidly gained traction. ${ }^{14-16}$ However, a recent Institute for Clinical and Economic Review report indicates less certainty regarding the effectiveness of this approach. ${ }^{14}$

Generally, community organizations either enroll eligible patients directly or partner with health care providers/ systems to identify and enroll patients. To the authors' knowledge, few health systems have implemented DPP within their operations-meaning that referrals, eligibility confirmation, and the actual program itself are provided within a health care system - and most existing programs implemented within health systems are of limited size. ${ }^{17}$ Most health systems implementing DPP continue to work with community partners and often rely on self-referrals in which patients request that their primary care provider (PCP) provides their latest hemoglobin A1c or diabetes risk score result to confirm eligibility. In some cases, health systems have implemented largescale community-facing programs, such as the Group Lifestyle Balance program offered by the University of Pittsburgh Diabetes Prevention Support Center. ${ }^{18}$

In March 2016, the Centers for Medicare \& Medicaid Services (CMS) announced that Medicare would begin to offer reimbursement for the delivery of the DPP starting in 2018. ${ }^{19}$ Because commercial providers often follow Medicare's reimbursement decisions, health care providers will be increasingly interested in how to offer this newly covered service for their patients using either fee for service or value-based payment for DPP reimbursement. Therefore, there will be substantial interest in implementing DPP, either with community partners or within a health care system.

Montefiore Health System (MHS) is a large and growing integrated delivery system, caring for $\sim 3$ million people in communities across the Bronx, Westchester, and the Hudson Valley, New York. MHS includes 11 hospitals, 190 ambulatory care sites, and has more than 28,000 employees. MHS has more than 140,000 inpatient discharges, 550,000 emergency room visits, and more than 4 million ambulatory care visits per year, and is almost $80 \%$ government payer. Within MHS is the Office of Community and Population Health $(\mathrm{OCPH})$, which houses the health education program. A health educator provides both individual and group coaching at 15 of 22 Montefiore Medical Group ambulatory primary care sites. Given the high prevalence of diabetes in the Montefiore patient population, preventing T2DM is a priority.

The purpose of this report is 2-fold: first, to describe the experience partnering with a community organization to implement DPP, and second, to discuss the process of fully implementing DPP within a large health delivery system.

\section{Community Partnership Model}

A time line of important program milestones and changes is provided in Figure 1. Additional details on dates of implementation are provided in the time line.

In late 2011, Montefiore's health education program partnered with the YMCA of Greater New York to offer the DPP to Montefiore patients. YMCA staff with experience delivering and training lifestyle coaches trained a subset of Montefiore health educators to teach the program. Initially, the DPP was offered to eligible patients at 4 ambulatory care sites, all of which were Federally Qualified Health Centers. In late 2014, efforts were made to expand and refresh the program by

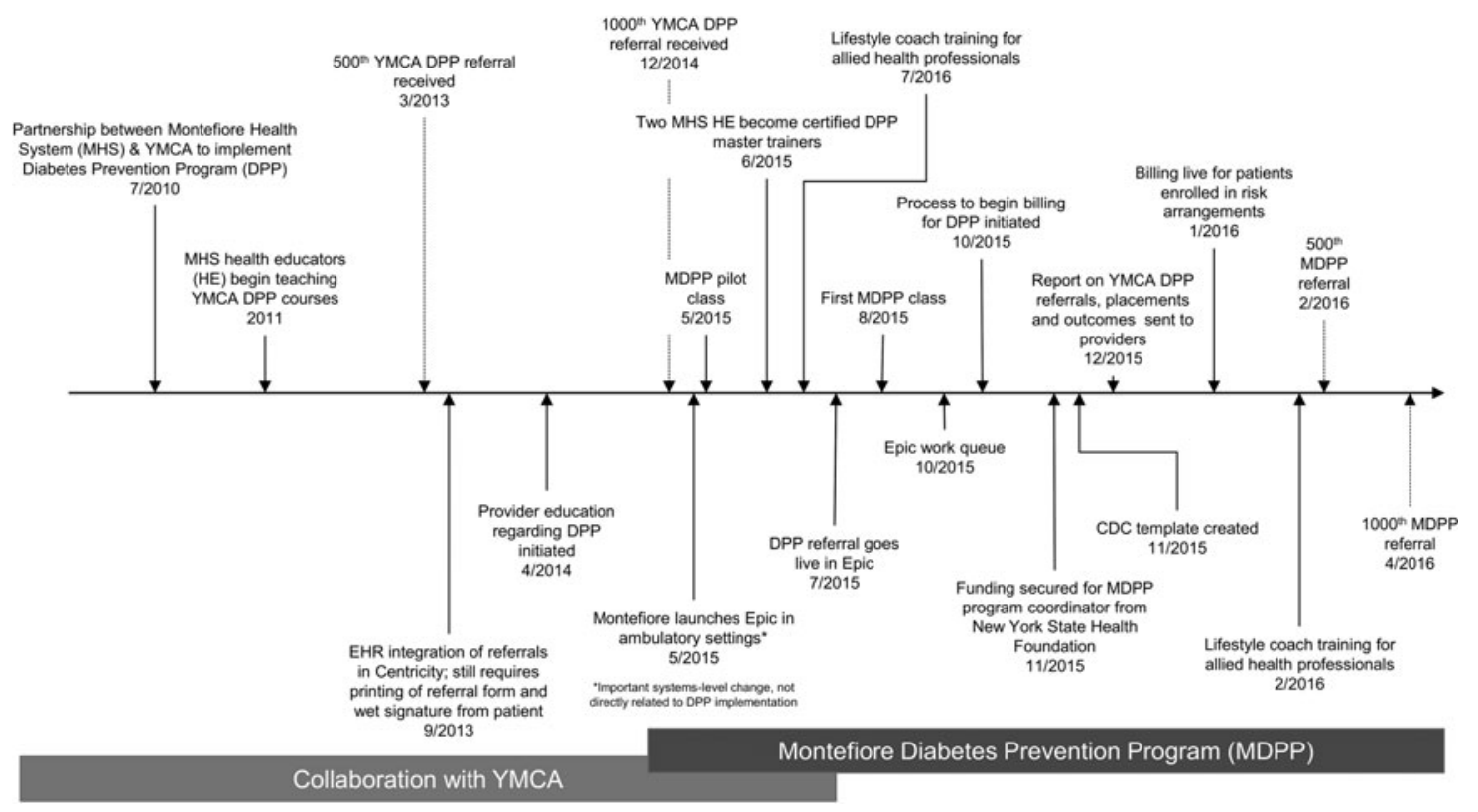

FIG. 1. Time line of DPP implementation, including collaboration with YMCA and integration within Montefiore Health System. EHR, electronic health record; YMCA, Young Men's Christian Association. 
offering it at more sites and doing extensive outreach with providers to alert them about the existence of the program.

Initially, referrals to the YMCA were generated on paper by PCPs, but subsequently were made available in the ambulatory electronic health record (EHR). For both referral methods, patients had to provide written consent to have their information (ie, demographics, insurance information, most recent A1c, height, weight, preferred language) sent to the YMCA. Referral forms were then faxed to a central number at the YMCA. YMCA staff created a random patient identifier, logged the referral into a spreadsheet, and attempted to reach the patient and place them in a class.

Patients were given the option of attending classes at local YMCAs (26\%), Montefiore ambulatory care centers $(62 \%)$, or other community settings such as churches $(12 \%)$, where trained lifestyle coaches led the classes. Classes were taught at 7 unique Montefiore locations, and most classes were taught by Montefiore health educators; the rest were taught by YMCA staff. For many of these classes, a majority of participants were Montefiore patients, but the classes could include non-Montefiore patients.

Class participation, weight, and physical activity logs were entered into MYnetico, a database used by the YMCA to transmit program participation data to the national YMCA data repository. Initially monthly (subsequently quarterly), YMCA staff would provide Montefiore with a de-identified line-level list of referrals, individuals who were scheduled into classes, and the first and last weight among participants who were "enrolled" (defined as those attending $\geq 3$ sessions). These data did not include any information about class attendance or weight at each class. If the patient did not attend $\geq 3$ classes, the patient would be disenrolled and YMCA staff would make additional outreach attempts to enroll the patient in a new class. Although the classes were not limited to Montefiore patients, the data received by Montefiore were limited to these patients.

The YMCA used patient insurance information to attempt to bill for the service, although reimbursement was limited to a very small number of payers (including a Center for Medicare and Medicaid Innovation grant involving Medicare patients).

\section{Opportunities and challenges in community partnership}

The collaboration with the YMCA was initiated for a number of reasons. First, the YMCA had an established track record of implementing the program and was a willing collaborator. Second, the YMCA agreed to manage the entire program after the referral was received, including scheduling patients and managing all program data. At the time of initiation, MHS did not have the internal capacity to manage these aspects of the program. The YMCA also trained MHS staff as lifestyle coaches and allowed the program to be taught at both Montefiore and community-based sites, increasing access to classes. The YMCA benefited from having an increased pool of lifestyle coaches, including those who could lead classes in Spanish.

As with the implementation of any large-scale and complex program, a number of challenges emerged. The first challenge was persuading Montefiore PCPs to agree to the referral workflow, which included obtaining written patient consent for the referral to be sent to the YMCA. In addition, despite the YMCA protocol of sending periodic patient updates to the referring providers, most providers reported that they did not receive these letters and did not know if any of their referred patients had been placed or how they were doing in the program. Referring providers indicated that receiving regular updates about the status of their patients in the DPP would be an important lever for ensuring future referrals.

With few insurers reimbursing for DPP, it was difficult to secure sufficient outreach, placement, and tracking resources to keep up with referral volumes. Likewise, there often was not sufficient revenue to cover the costs of patient materials (eg, calorie guides) or the trainers, even when Montefiore staff conducted the class. Despite these budgetary constraints, Montefiore and the YMCA agreed not to charge patients outof-pocket, given the economic realities of this generally lowincome and underserved patient population.

Financial constraints also contributed to the limited number of classes available to meet the needs of the patients, many of whom could not find classes at times and locations that would allow them to overcome barriers related to their work schedules, family commitments, and transportation challenges.

The final challenge was data availability. The YMCA program details were logged into MYnetico by the coaches, with de-identified summary data logged into an Excel spreadsheet (by YMCA staff) and provided to Montefiore on a quarterly basis. Without the ability to reidentify the patients participating in the program, Montefiore was not able to assess the overall success of the program with regard to weight loss, retention in the program, diabetes prevention, A1c control, and other related outcomes such as blood pressure. In addition, the lack of a feedback loop between DPP and outpatient providers made care coordination challenging. The provision of aggregateonly data also limited what information could be shared with referring providers to increase their likelihood of referring eligible and interested patients.

\section{In-System Implementation of the DPP}

Ultimately, as interest in the program increased along with internal resources, Montefiore decided to bring the program inhouse. Fully implementing the program within the health system allowed MHS to leverage more resources while maintaining control of the data, which enabled greater program oversight and evaluation. The decision to bring the program within the health system was dependent on increased resources to manage the day-to-day operations of the program, including managing referrals and placing patients into classes.

In May 2015, the CDC approved Montefiore's preliminary application to run DPP in-house (hereafter referred to as the Montefiore Diabetes Prevention Program [MDPP]). The referral was reconfigured into the new EHR (Montefiore transitioned from GE's Centricity to Epic [Epic Systems Corporation, Verona, WI] in May 2015), and written patient consent was no longer required, thereby eliminating the hassle of printing the referral. Referrals could be generated by providers as before, but in the new system, referrals were electronically routed to a central Epic work queue assigned to a newly hired MDPP coordinator. Use of paper in any form has been eliminated from MDPP.

\section{MDPP implementation}

The MDPP coordinator, or designee, confirms patient eligibility in the EHR, and then calls referred patients to confirm interest and register them for a class. In the second quarter of 
2016, approximately 10 referrals were received each business day. All Montefiore health educators and a subset of allied health professionals, including community health workers and registered dietitians, underwent DPP training by National DPP master trainers, and many are now teaching classes. In an effort to reach as many patients as possible, classes are offered both during the day and in the early evening.

After each class, participation data are entered by the coaches into Epic, including information on weight, whether the participant completed a food tracker, and the number of minutes of physical activity reported in the prior week. Reports generated from these Epic data have been created to track and monitor progress as well as to fulfill the CDC reporting requirements (Fig. 2).

\section{Billing for DPP}

Despite evidence of their cost-effectiveness, an important component of the sustainability of MDPP when being implemented by a health system is the generation of sufficient revenue to partially offset program costs. ${ }^{20} \mathrm{OCPH}$ worked with the Montefiore billing department to begin the process of securing reimbursement for these classes. Initially, the focus was on fee-for-service, managed care, and value-based contracts (including full-risk contracts). However, it became clear that convincing payers to reimburse for DPP would be a longer process (before the decision by CMS to reimburse for DPP), so OCPH decided to focus on seeking reimbursement from Montefiore for the patients in risk arrangements.

A reimbursement rate of $\$ 33.70-\$ 47.16$ per risk patient per session was negotiated (using rates paid for diabetes group classes as the closest proxy), depending on the type of contract (eg, Medicaid, Medicare). In-house revenue cycle and billing experts were consulted, and the DPP advocacy Current Procedural Terminology code of $0403 \mathrm{~T}$ was selected. ${ }^{21}$ This code is entered for each class, and the billing department sends a payment to the OCPH for all patients who qualify. For those patients who do not qualify because they are not a part of a risk arrangement, patient statements are suppressed and no one is billed for the service. Currently, patients in risk arrangements make up $\sim 40 \%$ of the Montefiore DPP patient volume, allowing for sufficient revenue to cover the variable costs of the program, but not yet the fixed costs such as outreach, training, and data collection.

\section{Necessary resources}

Bringing the program in-house took approximately 5-6 months of work, including configuring the EHR templates, designing the billing workflows, securing seed funding (provided by the New York State Health Foundation), hiring

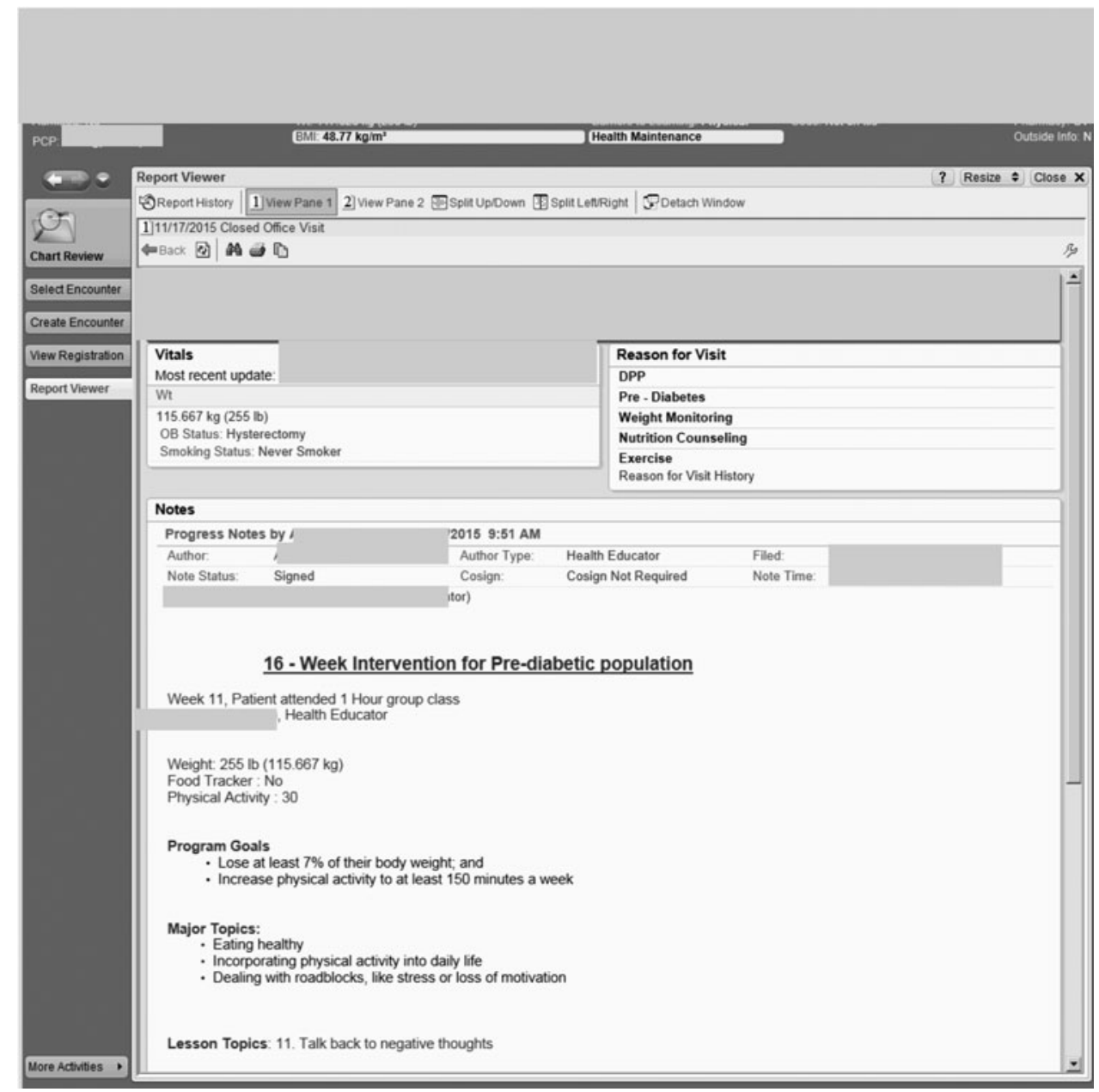

FIG. 2. Example of Epic screenshot tracking patient attendance. 
the MDPP coordinator (funded through a New York State Health Foundation grant), building the CDC reports, and creating the clinical referral workflows and training guides. It is important to keep in mind that this work was done simultaneously with the rollout of a new EHR system and may be more efficiently implemented in an environment with established informatics. Approximate details on the staffing requirements to sustain DPP at scale are provided in Table 1.

\section{Program Impact}

For analyses of both YMCA's DPP and MDPP tracking data, approval was obtained from the Albert Einstein College of Medicine or Montefiore Medical Center Institutional Review Boards.

The number of referrals to the YMCA DPP increased initially and was relatively stable from 2012 to 2015, starting with 8 (2011), 374 (2012), 354 (2013), 285 (2014), and increasing to 374 through the first half of 2015. The number of placements for these same years was 4 (2011), 59 (2012), 85 (2013), 63 (2014), and 76 (2015), although it is important to note that an individual referred in 1 year could be placed in subsequent years. The placement rate among Montefiore patients referred to the YMCA's DPP across implementation was $20.6 \%$.

After starting a pilot class in May 2015, 24 class cycles have been initiated by the MDPP through July 2016 and 282 patients have begun attending classes. More than 1250 referrals were made (after excluding duplicate referrals), and 282 patients were placed $(22.1 \%$ placement rate) in the first 11 months of MDPP implementation. Figure 3 summarizes the referral rate by quarter for the YMCA's DPP from quarter 12012 to quarter 22015 and compares the referral rate to the referral rate after the MDPP referral went live in the EHR. Referrals to MDPP have increased monotonically as more providers have been made aware of the program and the referral process has been streamlined. The training of site-specific allied health staff as lifestyle coaches with whom referring providers are familiar is another factor that may increase the number of referrals.

In addition, Montefiore Medical Group plans to add a measure of MDPP referrals to their quality measure set, enabling the medical leadership to see the aggregate MDPP referral volume (as a percent of total eligible patients) for each site. MHS plans to examine whether this systems-level factor impacts referral frequency. Although referrals to the MDPP have increased, it is important to note that, if approximately a third of more than 350,000 Montefiore adult patients have prediabetes, only a very small proportion of eligible patients are being referred to the program, indicating that there is still much opportunity for program growth.

The primary short-term quantifiable metric for DPP is weight change (ie, weight loss). In the YMCA's DPP, average weight loss was $3.4 \%$ among 287 patients attending

Table 1. Approximate Resources Needed to Implement and Sustain Diabetes Prevention Program in a Large Integrated Health Care Delivery System

\begin{tabular}{|c|c|c|}
\hline & $\begin{array}{l}\text { Estimated FTE } \\
\text { per position per } \\
\text { year or cost }\end{array}$ & Notes/tasks \\
\hline \multicolumn{3}{|l|}{ Administrative/management } \\
\hline High-level administrative & $0.025-0.05$ & Provide high-level oversight; strategic support \\
\hline Project manager & 0.10 & Assist with program implementation, coordination, and reporting \\
\hline Data support & 0.05 & $\begin{array}{l}\text { Higher range of FTE required if interest in conducting more } \\
\text { rigorous research/evaluation }\end{array}$ \\
\hline \multicolumn{3}{|l|}{ Program implementation } \\
\hline Health education director & 0.20 & $\begin{array}{l}\text { Directs MDPP, supervises MDPP coordinator and health } \\
\text { education managers }\end{array}$ \\
\hline DPP coordinator & 1.00 & $\begin{array}{l}\text { Reaches out to all referrals, schedules all classes, fills } \\
\text { in for lifestyle coaches }\end{array}$ \\
\hline $\begin{array}{l}\text { Health education managers } \\
\qquad(\times 2-3)\end{array}$ & 0.10 & $\begin{array}{l}\text { Health education managers teach classes but also provide } \\
\text { management support to health educators housed at } \\
\text { outpatient clinics }\end{array}$ \\
\hline Health educators $(\times 8-12)$ & $0.075-0.10$ & $\begin{array}{l}\text { Assumes each health educator teaches } 2 \text { class cycles per year } \\
(24 \text { sessions: } 16 \text { core }+8 \text { maintenance) and also spends } \\
\text { time documenting and preparing for classes }\end{array}$ \\
\hline Master training certification & $\begin{array}{l}\$ 1500-\$ 3500 \\
\text { per person }\end{array}$ & $\begin{array}{l}\text { One-time cost for master training certification; allows institution } \\
\text { to train its own lifestyle coaches }\end{array}$ \\
\hline \multicolumn{3}{|l|}{ Epic at Montefiore } \\
\hline $\begin{array}{l}\text { Epic consultants-template } \\
\text { creation }\end{array}$ & 0.025 & Resource needs may be higher during start-up of program \\
\hline Epic consultants-reporting & 0.025 & \\
\hline \multicolumn{3}{|l|}{ Other internal resources } \\
\hline Billing support & 0.025 & Resources to set up billing \\
\hline \multicolumn{3}{|l|}{ Outpatient clinics } \\
\hline Front desk staff & In-kind & Register patients \\
\hline Referring providers & In-kind & $\begin{array}{l}\text { Identify patients with prediabetes who may be interested } \\
\text { in participating in DPP }\end{array}$ \\
\hline
\end{tabular}

DPP, Diabetes Prevention Program; FTE, full-time employee; MDPP, Montefiore Diabetes Prevention Program 


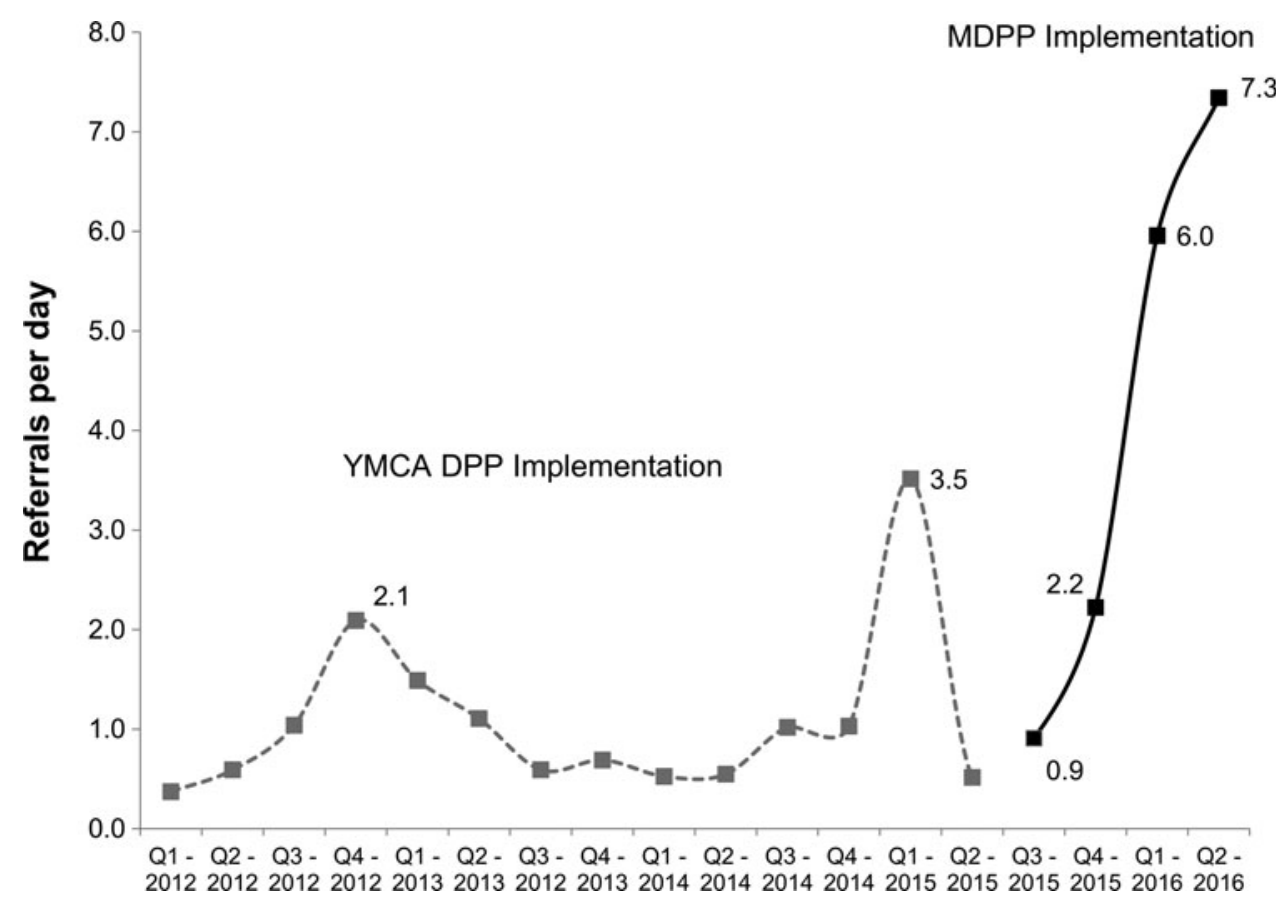

FIG. 3. Referral rate by quarter during the implementation of the YMCA's DPP compared to baseline implementation of the MDPP. DPP, Diabetes Prevention Program; MDPP, Montefiore Diabetes Prevention Program; Q, quarter.

$\geq 3$ classes. Among 61 graduates from the fall/summer 2015 wave of classes, average weight loss was $3.8 \%$ and $38 \%$ lost more than $5 \%$ of their initial body weight (data from the spring/summer classes are not yet available). Other inperson programs have observed average weight loss between $2.5 \%$ and $7 \%$, with most observing weight loss at $\sim 4.5 \%-6 \% .^{10,14,22-26}$ Observational data from the DPP trial suggest that diabetes risk reductions of about $35 \%-38 \%$ were observed at levels of weight loss between $0 \%-3 \%$ and $3 \%-5 \% .^{27}$ Furthermore, a monotonic trend toward increasing weight loss was observed among participants most engaged in the program; those attending $\geq 17$ sessions (ie, those attending at least 1 maintenance session) lost the most weight $(5.5 \%, n=17)$, consistent with other studies. ${ }^{24}$

\section{Lessons Learned}

Table 2 summarizes challenges and resources needed to overcome these challenges in internal implementation of DPP. A list of the most important lessons learned is provided below:

- Providers resist overly burdensome referral pathways. Integrating the referral documentation and transmission into the EHR and removing the need to print, scan,

Table 2. List of Challenges Implementing Diabetes Prevention Program Within an Integrated Health Care Delivery System and Resources and Opportunities for Program Improvement

Ongoing challenges

Increasing the percentage of providers who refer prediabetic patients to DPP

Reducing time between patient referral and class enrollment

Engaging special populations (eg, men, adolescents)

Class retention

DPP class reimbursement
Resources needed and opportunities for program improvement

Provision of provider performance feedback on rates of referral, enrollment status of their patients, and patient progress

Offer periodic training on referral process to providers

Inclusion of percent of patients with prediabetes referred to DPP in ambulatory care performance measures

Commitment to flexible staffing models allows for ongoing engagement with referred patients

Training of additional allied health staff as DPP lifestyle coaches

Offer adapted curriculum (ie, condensed classes, different class time line, virtual classes)

Use of peer leader model

Use EHR to capture patient barriers to enrollment and attendance (eg, transportation, childcare) in structured data fields and develop strategies to address barriers

Assess the impact of incentives to participation (eg, calorie counters, pedometers, MetroCards)

Where possible, create in-house reimbursement for patients in risk-based and/or capitated contracts

Medicare reimbursement for DPP starting in 2018 sets precedent for other payors

DPP, Diabetes Prevention Program; EHR, electronic health record. 
sign, or fax help save them time and increase the likelihood of referring patients. ${ }^{28}$

- Owning the referral queue in-house allows flexible staffing models; a dedicated coordinator, the coaches, or a supervisor can reach out to patients, thereby reducing delay from referral to scheduling. The experience working with the YMCA revealed that a short interval between referral to scheduling was a strong predictor of whether a patient attended $\geq 3$ classes, but was not associated with improved weight loss outcomes (E. Chambers, PhD, oral communication, June 6, 2016).

- Provider networks that manage risk are in a better position to create a funding stream for the DPP.

- It is critical to offer patients a wide variety of choices regarding class location, time of day, and language.
This requires a large pool of lifestyle coaches and the capacity to offer classes in numerous places. Having Montefiore employ master trainers who could provide training to allied health staff was crucial to support staff expansion as needed.

\section{Future Efforts}

With all operational aspects of this program now under Montefiore management, future efforts will be focused on training allied health staff (eg, registered dietitians, community health workers, and newly hired health educators) to increase systemwide capacity to offer DPP, piloting novel methods of content delivery (including concatenated class and virtual visits), assessing where and why patient participation drops,
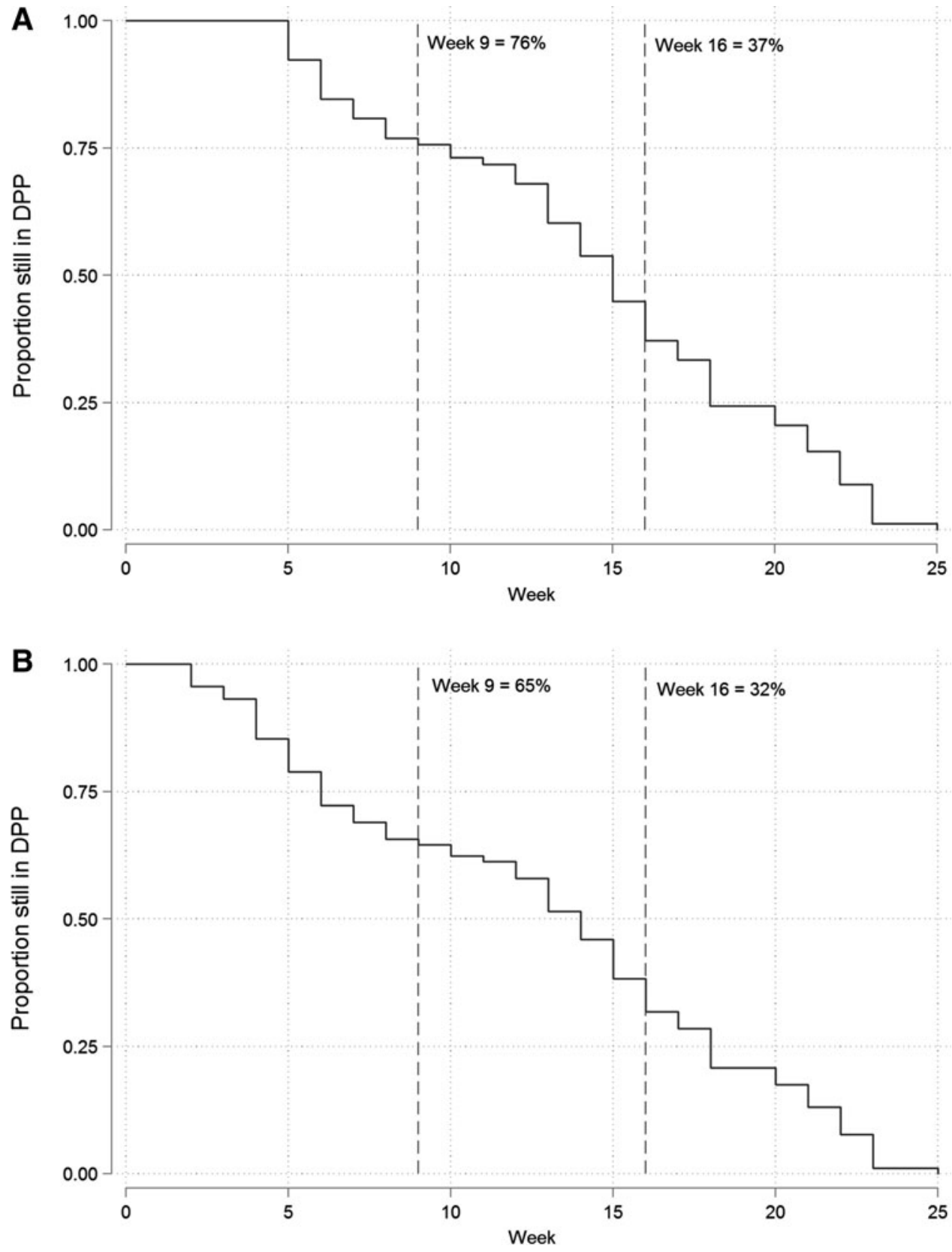

FIG. 4. Percent of Diabetes Prevention Program (DPP) participants still participating in the program by week. (A) Limited to patients attending the 4 th session and (B) among all participants. 
and developing data-driven approaches to improve patient adherence and outcomes. An example of how access to program data can inform programmatic approaches is shown in Figure 4, which displays the proportion of participants still engaged in the DPP by week among participants in the summer/fall MDPP wave. In this study, dropouts appear to occur fairly evenly throughout the program, suggesting that there is not a single week where program participation declines. Therefore, strategies to improve sustained engagement with the program should be tailored throughout the program.

Ensuring that providers continue to refer their eligible patients and providing provider performance feedback will be critical to the continued growth of the program. MHS plans to prepare periodic performance reports summarizing patient engagement with DPP (eg, whether they were enrolled in a class), number of classes attended, and program outcomes (eg, weight change). Given the integration of DPP tracking data in the EHR, plans are to conduct rigorous comparative effectiveness analyses comparing the weight change among participants to a matched set of nonreferred patients with prediabetes and nonenrolled referrals. In addition, because of data integration, there is an opportunity to track the long-term sustainability of intervention effects on both proximal (eg, weight change, glycemic control) and long-term outcomes (eg, incidence of T2DM).

In addition, it will be important to assess the effectiveness of intervening with patients at the lower end of the prediabetes glycemic range (A1c 5.7\%-5.9\%) versus those at the higher end $(6.0 \%-6.4 \%)$, particularly when resources are limited and the need is so great. Some have suggested limiting DPP eligibility and/or reimbursement to those at higher risk, ${ }^{29}$ although additional cost-benefit analysis will be needed to determine whether a threshold should exist. ${ }^{7}$

\section{Conclusions}

This experience shows that partnering with an external organization can help ease the introduction of DPP into a health system, but as the program expands, it may make sense to build internal capacity. DPP has been shown to work in a variety of different patient populations and it is possible for mid- to large-sized provider networks to offer it, using health educators or other allied health staff.

With reimbursement on the horizon, there is optimism that more health systems will implement and/or expand their DPP efforts. Nationwide implementation requires the use of additional delivery modalities (eg, telephonic or web-based counseling), as well as a much wider footprint for curriculum delivery outside the clinical setting, including in schools, faith-based organizations, and senior centers. In addition, because some individuals will not engage (or be able to engage) in a program like DPP, it is important that upstream approaches to increase access to and promotion of healthy food and active living be pursued in conjunction with health education programs. ${ }^{30}$

\section{Acknowledgments}

The authors would like to thank the YMCA of Greater New York for their years of partnership and collaboration; this project would not have been possible without their guidance and assistance. The authors also would like to thank the New York State Health Foundation for providing grant funding to support program coordination and patient outreach. The authors also would like to acknowledge the work of the Montefiore health educators and other lifestyle coaches who led the classes. Helpful comments from Elizabeth Walker, $\mathrm{PhD}$, Diane McKee, MD, and Earle Chambers, PhD, MPH, improved the article substantially.

\section{Author Disclosure Statement}

Drs. Rehm, Harris-Hollingsworth, and Parsons, Ms. Marquez, and Ms. Spurrell-Huss declared no conflicts of interest with respect to the research, authorship, and/or publication of this article. The authors received no financial support for this article.

\section{References}

1. Centers for Disease Control and Prevention. Crude and ageadjusted rates of diagnosed diabetes per 100 civilian, noninstitutionalized adult population, United States, 1980-2014. www.cdc.gov/diabetes/statistics/prev/national/figageadult.htm Accessed July 25, 2016.

2. Centers for Disease Control and Prevention; National Center for Health Statistics. Leading causes of death. www .cdc.gov/nchs/fastats/leading-causes-of-death.htm Accessed July 24, 2016.

3. Beckman JA, Creager MA, Libby P. Diabetes and atherosclerosis: epidemiology, pathophysiology, and management. JAMA 2002;287:2570-2581.

4. Tabak AG, Herder C, Rathmann W, Brunner EJ, Kivimaki M. Prediabetes: a high-risk state for diabetes development. Lancet 2012;379:2279-2290.

5. Institute for Health Metrics and Evaluation (IHME). GBD Compare. 2015. http://vizhub.healthdata.org/gbd-compare Accessed April 20, 2016.

6. Caballero AE. Metabolic and vascular abnormalities in subjects at risk for type 2 diabetes: the early start of a dangerous situation. Arch Med Res 2005;36:241-249.

7. The International Expert Committee. International Expert Committee report on the role of the A1C assay in the diagnosis of diabetes. Diabetes Care 2009;32:1327-1334.

8. Menke A, Casagrande S, Geiss L, Cowie CC. Prevalence of and trends in diabetes among adults in the United States, 1988-2012. JAMA 2015;314:1021-1029.

9. Bullard KM, Saydah SH, Imperatore G, et al. Secular changes in U.S. Prediabetes prevalence defined by hemoglobin A1c and fasting plasma glucose: National Health and Nutrition Examination Surveys, 1999-2010. Diabetes Care 2013;36:2286-2293.

10. Knowler WC, Barrett-Connor E, Fowler SE, et al. Reduction in the incidence of type 2 diabetes with lifestyle intervention or metformin. N Engl J Med 2002;346:393403.

11. Albright AL, Gregg EW. Preventing type 2 diabetes in communities across the U.S.: the National Diabetes Prevention Program. Am J Prev Med 2013;44(4 suppl 4):S346-S351.

12. Ackermann RT, Marrero DG. Adapting the Diabetes Prevention Program lifestyle intervention for delivery in the community: the YMCA model. Diabetes Educ 2007;33:69, 74-65, 77-68.

13. American Association of Diabetes Educators. AADE Diabetes Prevention Program. https://www.diabeteseducator.org/ practice/diabetes-prevention-program. Accessed June 20, 2016.

14. Institute for Clinical and Economic Review \& California Technology Assessment Forum. Diabetes Prevention Programs: 
Effectiveness and Value. May 2016. http://icer-review.org/ wp-content/uploads/2016/05/CTAF_DPP_Draft_Evidence_ Report_050916-1.pdf Accessed July 27, 2016.

15. Su W, Chen F, Dall TM, Iacobucci W, Perreault L. Return on investment for digital behavioral counseling in patients with prediabetes and cardiovascular disease. Prev Chronic Dis 2016;13:E13.

16. Smith KJ, Kuo S, Zgibor JC, et al. Cost effectiveness of an internet-delivered lifestyle intervention in primary care patients with high cardiovascular risk. Prev Med 2016;87: 103-109.

17. Hafez D, De Michele M, Sachdev N. Frontline account: resident-led implementation of the National Diabetes Prevention Program within primary care clinics of a large, academic medical center. J Gen Intern Med 2016;31:573-575.

18. Venditti EM, Kramer MK. Diabetes Prevention Program community outreach: perspectives on lifestyle training and translation. Am J Prev Med 2013;44(4 suppl 4):S339-S345.

19. Department of Health and Human Services, Centers for Medicare \& Medicaid Services. Certification of Medicare Diabetes Prevention Program. www.cms.gov/ResearchStatistics-Data-and-Systems/Research/ActuarialStudies/ Downloads/Diabetes-Prevention-Certification-2016-03-14 .pdf Accessed April 25, 2016.

20. Herman WH, Hoerger TJ, Brandle M, et al. The costeffectiveness of lifestyle modification or metformin in preventing type 2 diabetes in adults with impaired glucose tolerance. Ann Intern Med 2005;142:323-332.

21. Centers for Disease Control and Prevention. National Diabetes Prevention Program. Billing codes. www.cdc.gov/ diabetes/prevention/lifestyle-program/deliverers/biling.html Accessed July 15, 2016.

22. Ackermann RT, Finch EA, Caffrey HM, Lipscomb ER, Hays LM, Saha C. Long-term effects of a community-based lifestyle intervention to prevent type 2 diabetes: the DEPLOY extension pilot study. Chronic Illn 2011;7:279-290.

23. Bozack A, Millstein S, Garcel JM, Kelly K, Ruberto R, Weiss L. Implementation and outcomes of the New York
State YMCA diabetes prevention program: a multisite community-based translation, 2010-2012. Prev Chronic Dis 2014;11:E115.

24. Vojta D, Koehler TB, Longjohn M, Lever JA, Caputo NF. A coordinated national model for diabetes prevention: linking health systems to an evidence-based community program. Am J Prev Med 2013;44(4 suppl 4):S301-S306.

25. Brokaw SM, Carpenedo D, Campbell P, et al. Effectiveness of an adapted diabetes prevention program lifestyle intervention in older and younger adults. J Am Geriatr Soc 2015;63:1067-1074.

26. Katula JA, Vitolins MZ, Morgan TM, et al. The healthy living partnerships to prevent diabetes study: 2-year outcomes of a randomized controlled trial. Am J Prev Med 2013;44(4 suppl 4):S324-S332.

27. Maruthur NM, Ma Y, Delahanty LM, et al. Early response to preventive strategies in the Diabetes Prevention Program. J Gen Intern Med 2013;28:1629-1636.

28. Chambers EC, Wylie-Rosett J, Blank AE, et al. Increasing referrals to a YMCA-based diabetes prevention program: effects of electronic referral system modification and provider education in federally qualified health centers. Prev Chronic Dis 2015;12:E189.

29. Davidson MB, Kahn RA. A reappraisal of prediabetes. J Clin Endocrinol Metab 2016;101:2628-2635.

30. Wing RR, Goldstein MG, Acton KJ, et al. Behavioral science research in diabetes: lifestyle changes related to obesity, eating behavior, and physical activity. Diabetes Care 2001;24:117-123.

Address correspondence to: Amanda S. Parsons, MD, MBA Office of Community \& Population Health Montefiore Health System Bronx, NY 10467

E-mail: aparsons@montefiore.org 